\title{
Redes sociales de tabaquismo, consumo de alcohol y obesidad en adolescentes escolarizados de la ciudad de Lobos
} Smoking, alcohol consumption and obesity in a social network of
adolescents attending school in the city of Lobos, Buenos Aires

\author{
Dr. Andrés H. Mulassia , Dr. Raúl A. Borraccib, Dr. José G. E. Calderón ${ }^{a}$, \\ Dr. Patricio Vinay y Dr. Maximiliano Mulassia
}

\section{RESUMEN}

Introducción. El objetivo fue estudiar las estructuras de distintas redes sociales de adolescentes con respecto a la presencia de tabaquismo, consumo de alcohol y obesidad, para conocer las vías de influencia que pudieran ejercer las amistades sobre el mantenimiento o cesación de estos hábitos.

Material y métodos. Mediante una encuesta y mediciones directas se realizó un estudio observacional y transversal en 296 adolescentes de entre 14 y 18 años de una escuela de la provincia de Buenos Aires. Se obtuvo información sobre parámetros antropométricos, tabaquismo, consumo de alcohol y sobre las relaciones de amistades entre los encuestados, a fin de construir las redes sociales.

Resultados. El 18,8\% de las mujeres y el 16,5\% de los varones fumaban, el $23,3 \%$ y el $39,4 \%$ consumían alcohol y el 15,3\% y el 19,0\% presentaban sobrepeso, respectivamente. Las redes construidas mostraron que tanto los tabaquistas comolos consumidores de alcohol tenían un alto grado de conexión y centralidad en la red. El análisis de afinidad demostró que la formación de conglomerados favorece el contacto entre quienes tienen los mismos hábitos.

Conclusiones. El estudio de redes sociales de adolescentes permitió conocer las relaciones de afinidad entre quienes presentaban hábitos de consumo de tabaco o alcohol, u obesidad. Tanto los fumadores como los bebedores parecen estar estrechamente enlazados en la red, mientras que los obesos ocuparían una ubicación más marginal. El conocimiento de la conformación de las redes sociales y la intervención sobre sólo algunos de sus actores podrían usarse para propagar conductas saludables entre los adolescentes. Palabras clave: adolescencia, tabaquismo, alcohol, obesidad, redes sociales.

http:/ /dx.doi.org/10.5546/aap.2012.474

\section{INTRODUCCIÓN}

El tabaquismo, el alcoholismo y la obesidad en los adolescentes constituyen un problema de índole individual y social. Los hábitos adquiridos en la adolescencia suelen mantenerse en la adultez y son difíciles de modificar.
Por ejemplo, el estudio HABITS demostró que los niños que maduraban tempranamente tenían más riesgo de adquirir conductas poco saludables. ${ }^{1}$ Por su parte el GAZEL mostró la asociación entre tabaquismo, alcohol y drogadicción en jóvenes franceses. ${ }^{2}$ Además, se ha observado que si bien los adolescentes tienen conocimiento sobre los riesgos que suponen el consumo de tabaco o alcohol y el sobrepeso, sus hábitos parecen contradecirlo. ${ }^{3}$ El rol de las redes sociales en el mantenimiento y refuerzo de estos hábitos ha sido estudiado recientemente en adultos por Christakis y Fowler, 4,5 quienes demostraron la importancia de los lazos sociales en el comportamiento, al evaluar la dispersión del tabaquismo y la obesidad en una gran red social. Los vínculos creados en las redes sociales parecen influir en el comportamiento de los individuos cuando optan por fumar o cuidar su peso. ${ }^{6,7}$ Tener amistades que fuman, toman o son obesas aumentaría la tolerancia a estos hábitos e incrementaría la posibilidad de adoptarlos.

Fundamentalmente, una red social consta de un conjunto de personas que interactúan en un medio determinado, representados en un gráfico como una colección de puntos y vectores interconectados. ${ }^{8-10}$

En un estudio reciente en adolescentes, se halló que tener un amigo fumador o consumidor de alcohol, más que un familiar, aumentaba entre 4 y 10 veces la probabilidad de que el adolescente fuera fumador o bebedor. ${ }^{11}$ Esta influencia de los pares fue observada con anterioridad, ${ }^{7}$ pero no 
existen estudios que hayan analizado la topología de las redes sociales de fumadores, bebedores $\mathrm{u}$ obesos entre adolescentes del país. El estudio de estas redes estaría justificado ante el fracaso de otras estrategias de prevención implementadas previamente. El objetivo fue estudiar las estructuras de distintas redes sociales de adolescentes con respecto a la presencia de tabaquismo, consumo de alcohol y sobrepeso u obesidad, a fin de obtener información sobre las vías de influencia que pudieran ejercer las amistades respecto del mantenimiento o cesación de estos hábitos.

\section{MATERIAL Y MÉTODOS}

En abril de 2010 se realizó un estudio observacional y transversal en una muestra de adolescentes de una escuela pública urbana de la ciudad de Lobos, Buenos Aires, Argentina, con el propósito de obtener información sobre sus parámetros antropométricos y clínicos, hábitos alimentarios, tabaquismo y consumo de alcohol. Mediante una encuesta autoadministrada y la medición directa de algunos parámetros clínicos se relevaron 296 alumnos de 14 a 18 años que cursaban el Educación General Básica (EGB) y polimodal (de $2^{\circ}$ a $5^{\circ}$ años).

Las variables demográficas consideradas fueron: edad, sexo y año que cursa. Entre los hábitos alimentarios se preguntó sobre el cuidado para mantener o bajar de peso. Para evaluar tabaquismo se indagó sobre consumo y frecuencia, y para estimar las diferentes prevalencias se lo definió de dos maneras: por un lado, en forma conservadora, asumiendo el consumo de al menos un cigarrillo en los últimos 6 meses; por otro, se optó por el consumo diario de al menos un cigarrillo. Para evaluar la conducta con respecto al alcohol se preguntó sobre consumo y frecuencia. Se definió consumo de alcohol como su ingesta al menos 3 veces al año. No se recabaron datos sobre el perfil socioeconómico. Previa instrucción sobre el llenado del cuestionario, lo completaron los propios alumnos en presencia de los investigadores. La medición directa de los parámetros antropométricos y clínicos estuvo a cargo de los investigadores del estudio y constó de: peso, talla, índice de masa corporal (IMC), circunferencia de cintura y tensión arterial. Para definir sobrepeso y obesidad se utilizaron las recomendaciones del Consenso sobre factores de riesgo de enfermedad cardiovascular en pediatría de la Sociedad Argentina de Pediatría, ${ }^{12}$ y se consideró un IMC entre 25 y 30 para sobrepeso y mayor de 30 para obesidad. La determinación del aumento de la circunferencia de cintura como índice de grasa corporal, se basó en los criterios del mismo Consenso, asumiendo el punto de corte en el percentilo 90 según sexo y edad. ${ }^{12}$

\section{Construcción de las redes sociales de amistad}

En la misma encuesta se solicitó a cada alumno que indicara los nombres y apellidos de sus mejores amigos, para lo cual contaban con la posibilidad de anotar desde ninguno hasta diez nombres. Sólo se permitieron incluir amigos dentro del grupo de compañeros de la escuela, a fin de conocer los hábitos de los encuestados directamente; lo que no hubiese sido posible de incluirse amigos externos al curso. A cada encuestado se le adjudicó un número de orden para identificarlo, y se consideró a cada individuo un nodo, y a cada una de sus amistades una conexión hacia un nuevo nodo. Si el alumno era fumador, consumía alcohol o presentaba sobrepeso u obesidad, se lo marcaba con un color distinto a fin de poder identificarlo en la red. Con estos datos se construyeron y analizaron sendas redes para tabaquismo, alcohol y obesidad mediante el uso del algoritmo Kamada-Kawai ${ }^{13}$ en el software Pajek. ${ }^{14}$ Para la construcción y análisis de las redes se definieron distintos parámetros resumidos en el Apéndice (en versión electrónica).

\section{Análisis estadístico}

Los valores se expresaron como porcentajes o media \pm desvío estándar (DE). Para las comparaciones estadísticas se calculó el cociente de probabilidades (odds ratio, OR) e intervalo de confianza del $95 \%\left(\mathrm{IC}_{95 \%}\right)$ y las correspondientes pruebas de $\chi^{2}$, Fisher o McNemar. Las variables continuas se compararon con la $t$ de Student o Mann-Whitney, previa comprobación de su distribución con Kolmogorov-Smirnov. Todas las pruebas fueron bilaterales. Por tratarse de un muestreo poblacional descriptivo, se calculó el tamaño de la muestra con una confianza de $95 \%$, unas proporciones estimadas de consumo de tabaco y alcohol y de sobrepeso de por lo menos $15 \%$, y un error máximo admisible de $4 \%$. El tamaño calculado fue de 306 individuos. Se utilizaron los paquetes informáticos Epi Info $2002^{\circledR}$ y SPSS $11.5^{\circledR}$.

Se obtuvieron los permisos de la autoridad municipal local y de la Inspección General de Escuelas, además del consentimiento de los alumnos, quienes podían aceptar participar o no. No se obtuvo consentimiento de los padres, pero fueron informados. 


\section{RESULTADOS}

De los 296 alumnos encuestados, se obtuvieron datos completos de 285 (96,3\%). La edad (media) fue $16,1 \pm 1,10$ años y el $61,8 \%$ fueron mujeres. El
$18,8 \%$ de las mujeres y el $16,5 \%$ de los varones fumaban al menos un cigarrillo diario (OR: 0,86; IC95\%: 0,43-1,68; $p=0,632)$, mientras que se elevó a $30,7 \%$ y $27,5 \%$ cuando se preguntó si habían fu-

FIGURA 1. Estructura de las distintas redes sociales de adolescentes de acuerdo a los hábitos de tabaquismo (a), consumo de alcohol (b) y la presencia de sobrepeso u obesidad (c). Los puntos más oscuros en las redes señalan la presencia de la condición en el individuo. Las redes de la izquierda corresponden a la población completa de adolescentes, mientras que las de la derecha se han simplificado para mostrar solo las relaciones de quienes tienen la condición de fumador, consumidor de alcohol o sobrepeso u obesidad

a. Red social de fumadores, completa y simplificada. Los círculos oscuros más pequeños corresponden a los individuos que fumaron en los últimos 6 meses, mientras que los más grandes a los que fuman diariamente.
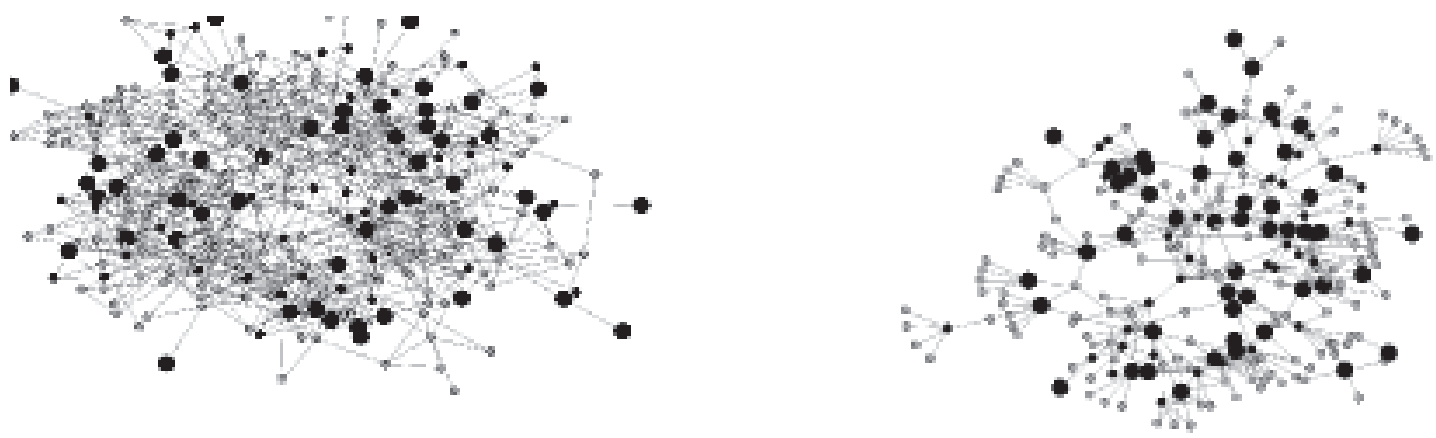

b. Red social de consumidores de alcohol (por lo menos 3 veces al año), completa y simplificada.
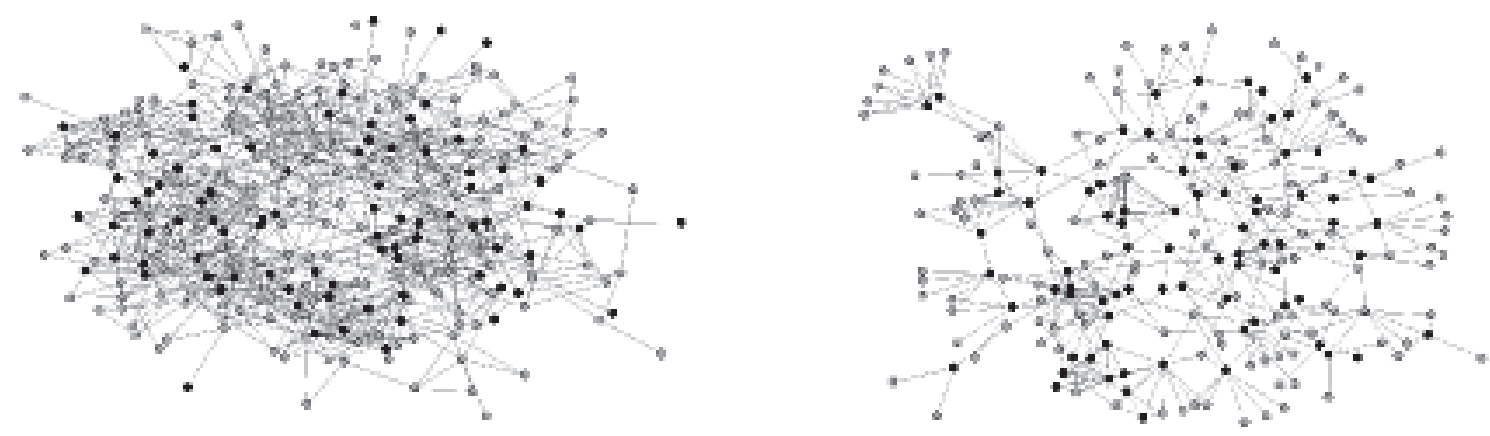

c. Red social de individuos con sobrepeso y obesidad, completa y simplificada. Los círculos oscuros más pequeños corresponden a los individuos con sobrepeso, mientras que los más grandes a los alumnos con obesidad.
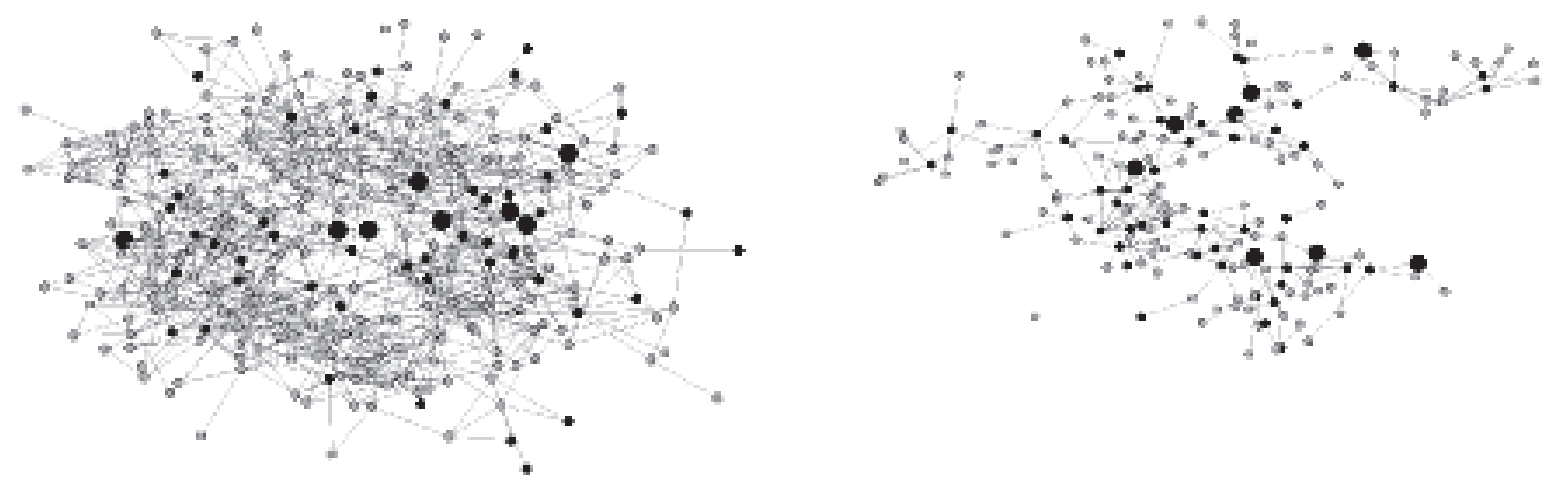
mado al menos un cigarrillo en los últimos 6 meses (OR: 0,86; IC95\%:0,49-1,50; $\mathrm{p}=0,570$ ).

El consumo de alcohol de 3 o más veces en el año se presentó en $39,4 \%$ de los varones y 23,3\% de las mujeres (OR: 2,15; IC95\%:1,24-3,73; $\mathrm{p}=0,004)$. Al asociar estas últimas dos variables sin discriminar por sexo, el 8,4\% de los adolescentes fumaba diariamente y consumía alcohol 3 o más veces al año.

El 15,3\% de las mujeres y el 19,0\% de los varones presentaban sobrepeso (OR: 0,77; IC95\%: $0,39-1,53 ; \mathrm{p}=0,418)$; mientras que la obesidad fue $3,5 \%$ y $1,9 \%$, respectivamente (OR: 1,88 ; IC95\%: $0,34-13,77 ; p=0,714)$. El IMC fue 22,1 $\pm 3,57$ en las mujeres y $21,9 \pm 3,33$ en los varones $(p=0,035)$; mientras que la circunferencia de cintura promedió los 79,8 8 9,81 cm en las mujeres y 81,6 6 9,43 cm en los varones $(\mathrm{p}=0,144)$. El 36,9\% de las mujeres y el $24,8 \%$ de los varones refieren cuidar su peso (OR: 1,78; IC95\%: 1,01-3,14; p=0,033). No se halló asociación en las mujeres entre el tabaquismo y el cuidado del peso $(p=0,1525)$, habida cuenta de la posibilidad de que algunas mujeres fumen para cuidar el peso (el 6,8\% cuidaba el peso y fumaba).

Las cifras de tensión arterial sistólica fueron respectivamente para las mujeres y los varones 109,1 \pm 14,95 mmHg (mínimo: 80, máximo: 160) y 115,9 $\pm 14,27$ mmHg (mínimo: 90, máximo: 170) ( $\mathrm{p}=0,0002)$. Las cifras de la tensión diastólica respectivas para mujeres y varones fueron 62,5 \pm 11,74 mmHg (mínimo: 40, máximo: 100) y 66,1 \pm 10,39 mmHg (mínimo: 40, máximo: 90) $(\mathrm{p}=0,007)$.

FIGURA 2. Gráficos de caja con los valores de centralidad de los individuos de cada red (centrality-closeness), de acuerdo a la condición de consumir o no tabaco o alcohol, o de tener o no sobrepeso u obesidad. Los valores de centralidad más altos corresponden a un mayor grado de conectividad en la red y los más bajos a un mayor aislamiento en ella. La distribución de los datos es no gaussiana y la prueba comparativa U de Mann-Whitney no mostró diferencias entre los grupos

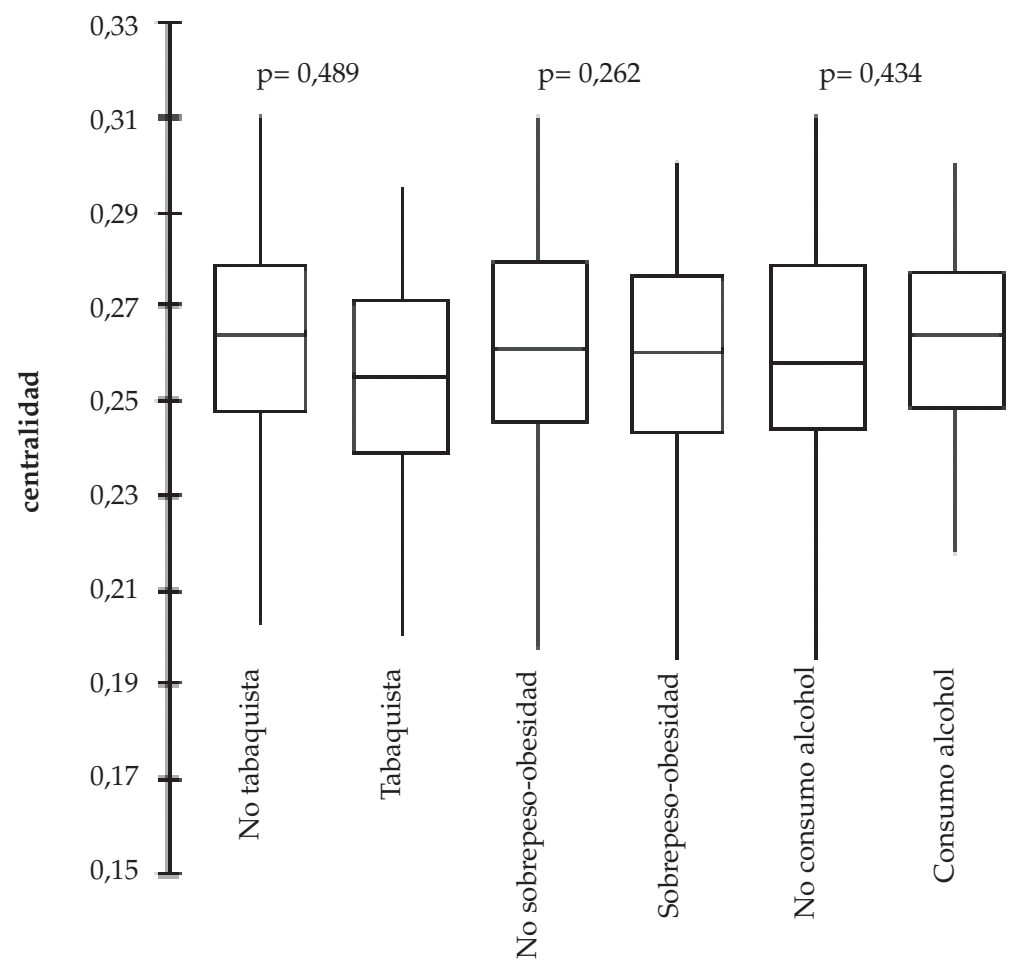




\section{Redes sociales}

En la Figura 1 se muestran las redes construidas a partir de los datos de tabaquismo, consumo de alcohol y sobrepeso y obesidad. En la secuencia gráfica de la izquierda se observa la estructura de las redes con los individuos que presentaban la condición estudiada (nodos oscuros) y aquellos que no fumaban, bebían, o que no presentaban sobrepeso u obesidad (nodos claros); mientras que las líneas representan las relaciones de amistad. En la secuencia de gráficos de la derecha se graficaron solo los individuos fumadores, bebedores u obesos junto con sus amistades, excluyendo aquellos adolescentes que no conformaban el grupo de sus amistades. La red completa presentó un diámetro de 9 y una distancia promedio de separación de 3,90. Las redes simplificadas de solo fumadores, consumidores de alcohol y obesos con sus amistades tuvieron los siguientes valores: 15 y 6,16; 11 y 5,01 y 16 y 7,03 , respectivamente.

Los valores de centralidad de los individuos de cada red, de acuerdo a la condición de consumir o no tabaco o alcohol, o de tener o no sobrepeso $\mathrm{u}$ obesidad se expresan en medianas y cuartilos en la Figura 2. El mayor valor de centralidad lo tuvieron los tabaquistas (media: 0,261, IC95\%:0,256-
0,266), mientras que el menor valor lo obtuvieron los que presentaban sobrepeso u obesidad (media: 0,250, IC95\%: 0,239-0,261). Por su lado, el análisis del número promedio de conexiones (grado de conexión) de los adolescentes de cada grupo mostró los siguientes valores: no tabaquistas $11,1 \pm 5,46$ contra tabaquistas $10,2 \pm 4,64(\mathrm{p}=0,139)$; no bebedores 10,6 $6 \pm 5,22$ contra consumidores $11,5 \pm 5,30$ $(\mathrm{p}=0,176)$ y no obesos $11,1 \pm 5,23$ contra obesos $9,6 \pm 5,19(\mathrm{p}=0,043)$, lo que demuestra un mayor aislamiento de estos últimos en la red.

En la Figura 3 se muestra la red de adolescentes fumadores diarios con sus amistades (red simplificada), señaladas en el gráfico de acuerdo al sexo. Se observa la formación de conglomerados de amigos, con predilección por el mismo sexo. También se destacan pequeños conglomerados de entre 2 y 5 individuos, no ligados al componente gigante de la red. El coeficiente $\mathrm{C}$ de aglomeración de la red completa fue $0,410 \pm 0,236$ y el teórico calculado para una red al azar sería $0,003(\mathrm{p}<0,001)$.

El grado de homofilia o afinidad por personas con la misma condición de fumador, bebedor u obeso se representa en la Figura 4 como la probabilidad de tener un amigo que fuma, bebe o es obeso. En todos los casos se observa que un fuma-

FIGURA 3. Red simplificada de adolescentes fumadores diarios en la que se observa la formación de conglomerados (clusters) de acuerdo al sexo ( $m=$ mujeres; $v=$ varones). Los conglomerados de mujeres se marcaron con línea punteada y fondo gris, $y$ los de los varones con línea continua y fondo blanco; se destaca la afinidad (relación de amistad referida) por género

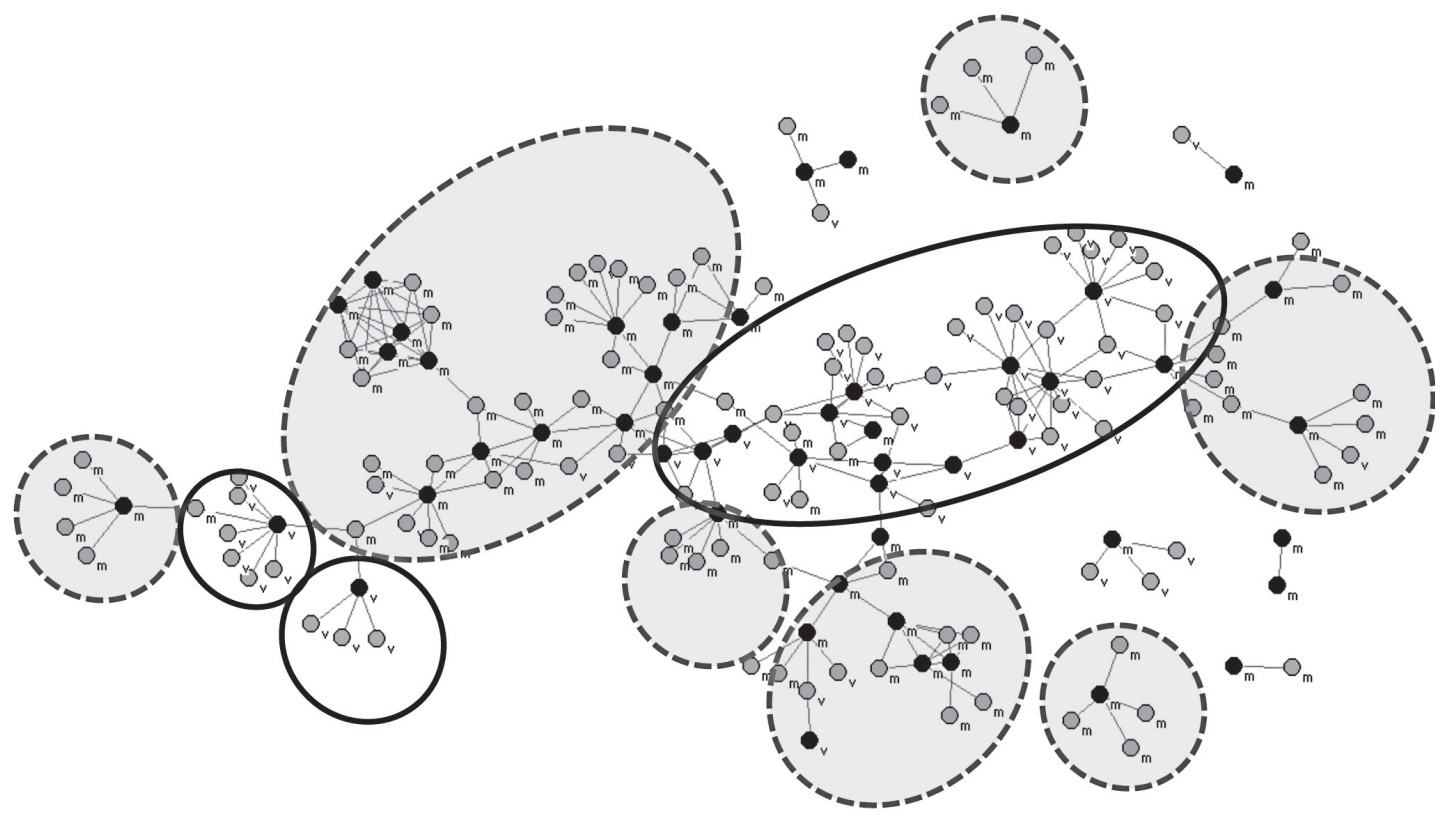


dor, bebedor u obeso tiene mayor probabilidad de relacionarse con un adolescente con la misma condición, que en el caso de los no fumadores, no bebedores o con peso normal.

En la Figura 5 se representó la evolución de una red de acuerdo al criterio que se use para definir consumo de alcohol. Ya que el consumo esporádico es muy frecuente, las redes consecutivas muestran cómo crece la densidad de bebedores (nodos oscuros) a medida que se flexibiliza la definición de consumidor; la secuencia de gráficos de la derecha individualiza la proporción de bebedores de acuerdo a la edad en cada red.

\section{DISCUSIÓN}

La interacción que se da dentro de una red permite el desarrollo y contagio de hábitos que se repiten por imitación o afinidad. ${ }^{4}$ En este es- tudio, la presencia de adolescentes fumadores o bebedores podría modificar el umbral de aceptación de estos hábitos y la eventual adquisición de ellos al aceptar compartir cigarrillos o bebidas. Para el caso de la obesidad, podría existir un mecanismo similar, aunque el problema tendría más componentes genéticos, culturales o de moda. Al comparar la prevalencia de tabaquismo en este estudio con la observada en una población similar, con un promedio de edad dos años menor (16,1 contra 13,8 años), se constató un incremento desde $6,8 \%$ a casi tres veces más en los varones (18,8\%). ${ }^{11}$ Comparado con el mismo estudio, la prevalencia de consumo de alcohol no mostró diferencias, lo que señalaría que el hábito de consumo podría instalarse precozmente. Con respecto al sobrepeso y la obesidad, las cifras halladas son ligeramente inferiores a las comu-

FIgURA 4. Gráficos de caja con los valores de homofilia (homophily) expresados como probabilidad de tener un amigo fumador, con sobrepeso u obesidad o consumidor frecuente de alcohol, de acuerdo a la condición de consumir o no tabaco o alcohol, o de tener o no sobrepeso u obesidad. Los valores más altos corresponden a una mayor probabilidad de tener un contacto con la condición estudiada. La distribución de los datos es no gaussiana y la prueba comparativa U de Mann-Whitney mostró diferencias entre los grupos; es decir que los tabaquistas tienen una mayor probabilidad de tener un amigo fumador que un no tabaquista, siendo lo mismo para la obesidad y el consumo de alcohol

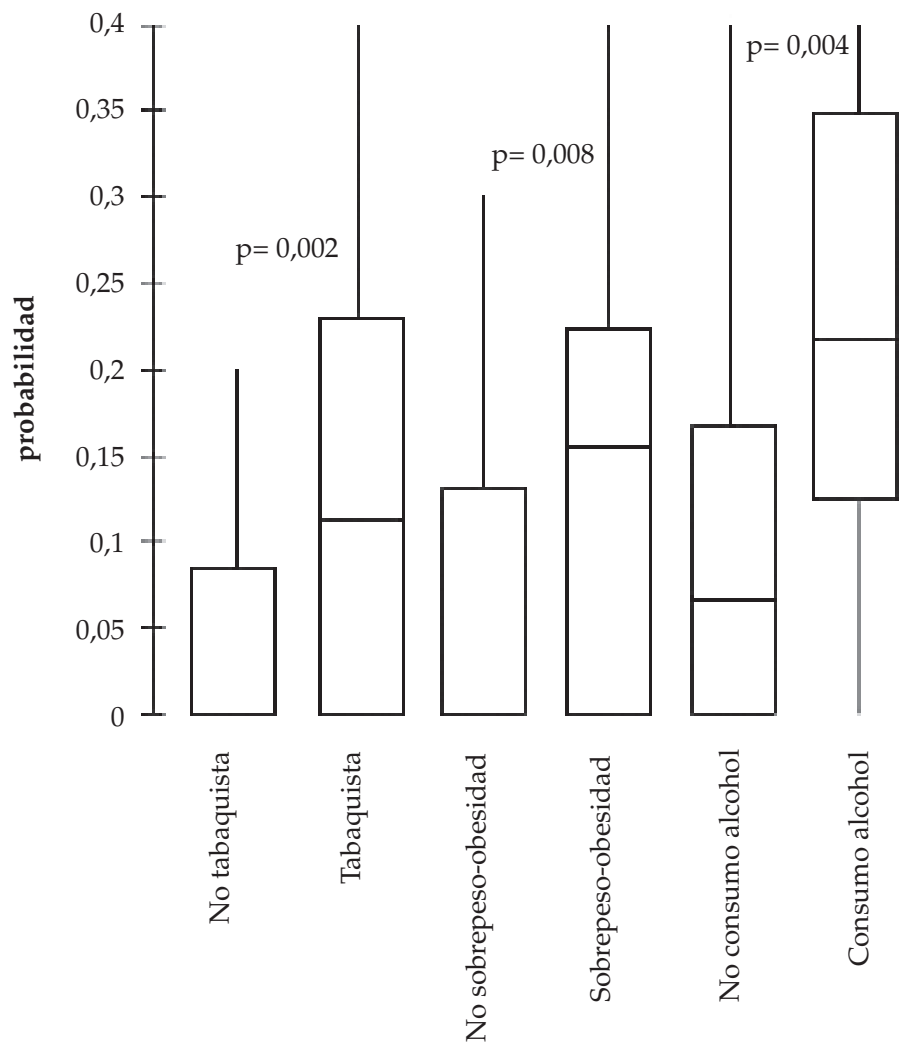


nicadas por Kovalskys y cols., ${ }^{15}$ en una encuesta nacional de hace 10 años y para la misma edad.

En contraposición a lo que ocurre con los adultos, en cuyas redes sociales los fumadores ocupan la periferia de la red, ${ }^{5}$ en los adolescentes, la ubicación de los fumadores es tan central como la de los no fumadores (centralidad y grado de conexión), lo que demuestra la tolerancia de los jóvenes a la presencia de tabaquistas en su comunidad. Los consumidores de alcohol también se distribuyen dentro de la red como si no lo fueran, lo que señala que son aceptados sin inconvenientes. Por el contrario, se observó un menor grado de conexión de los adolescentes con sobrepeso u obesidad $(9,6$ contra $11,1 \mathrm{p}=0,043)$ lo que indica un mayor aislamiento de estos últimos en la red. Este proceso de marginación de los adolescentes con sobrepeso ya fue corroborado por otros autores. ${ }^{16}$

Una característica observada es la tendencia a formar conglomerados de afinidad de acuerdo al sexo, con mayor probabilidad de que las mujeres por un lado y los varones por otro, conformen grupos de amistad. Por ejemplo, aunque no fue demostrado en este estudio, las mujeres suelen referir que fuman para controlar el peso, y no así los varones. ${ }^{17}$

El análisis de afinidad entre adolescentes con

Figura 5. Evolución de la red de adolescentes de acuerdo al consumo de alcohol: una vez al año (círculos oscuros pequeños), dos veces al año (círculos medianos) y tres o más veces al año (círculos grandes), junto con los porcentajes de consumidores de acuerdo a la edad. La secuencia de redes va ampliando la definición de consumo desde por lo menos 3 veces al año (a), por lo menos 2 veces al año (b) y consumidores independientemente de la frecuencia anual (c)

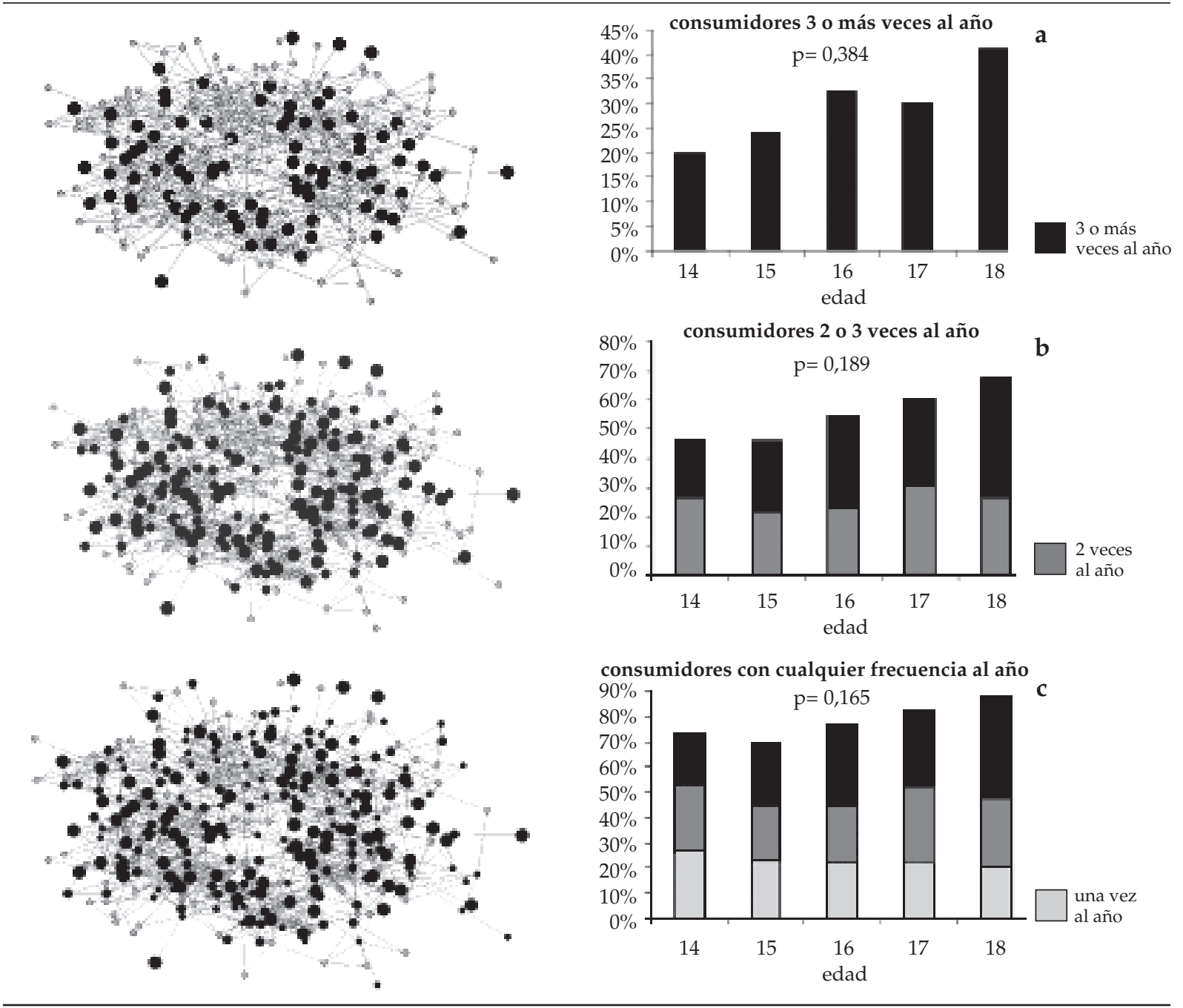


el mismo hábito (homofilia) mostró que un fumador tiene más probabilidades de tener un amigo que fuma, mientras que un no fumador tiene menos probabilidades de relacionarse con un tabaquista. La misma relación de probabilidades se halló en los consumidores de alcohol y en obesos. Igualmente, debe considerarse que los adolescentes con hábitos afines podrían estar expuestos a un mismo medio que los hace obesos o consumidores de tabaco o alcohol, y que la presunta relación de homofilia fuera el resultado de la covariación de este comportamiento. ${ }^{18}$

Christakis y cols. ${ }^{4}$ sugirieron que el contacto social entre adultos constituye un factor de difusión de la obesidad, más que la exposición a otros factores ambientales. En los adolescentes, los hallazgos sugieren que aquellos con sobrepeso $\mathrm{u}$ obesidad tienden a ocupar un lugar menos central dentro de las redes y suelen tener menos amistades, aislamiento que requeriría un abordaje epidemiológico diferente. De todas formas, la homofilia mostró también que los obesos suelen tener mayor afinidad por amigos con la misma condición de peso; o sea, que la intervención sobre unos podría afectar la condición de los otros.

Como ha sido comunicado, si la cesación de fumar tiene un efecto de difusión de persona a persona, la intervención sanitaria sobre algunos pocos adolescentes fumadores con un alto grado de conexión en la red, podría multiplicar el efecto deseado a sus amigos en forma de cascada. ${ }^{19} \mathrm{Un}$ efecto similar podría esperarse para las intervenciones orientadas a reducir el consumo de alcohol. Así, los fenómenos de las redes podrían explotarse para difundir conductas saludables entre los adolescentes. ${ }^{20-22}$

Entre las limitaciones de este estudio se debe considerar que el intervalo de edad podría ser demasiado amplio para plantear amistades entre los grupos; lo mismo ocurriría para las amistades entre varones y mujeres. Los lazos sociales entre adolescentes podrían ser más lábiles y cambiantes, modificando en poco tiempo la arquitectura de la red. Aunque la direccionalidad de la amistad (cuando uno se atribuye la amistad de otro, que a su vez no lo considera amigo) parece tener influencia en la adquisición del hábito de fumar o en tener sobrepeso ${ }^{4-5}$ no fue considerada en nuestro estudio, a causa del tamaño de la muestra. Otra limitación es que los índices de obesidad no están basados en el IMC, edad y puntaje Z, aunque deseamos aclarar que el presente trabajo se realizó en abril de 2010 y en ese momento empleamos el Consenso de SAP de 2005. ${ }^{12}$ Posterior- mente, en 2011, se publicó la guía del Comité de Nutrición de SAP sobre obesidad, ${ }^{23}$ donde se recomendaba el puntaje Z de la OMS. De todas formas evaluamos las diferencias entre nuestra metodología y la del puntaje Z. Como la presente muestra estaba compuesta por adolescentes de entre 14 y 18 años, la comparación de los límites 25-30 y $>30$ para sobrepeso y obesidad con los puntajes $Z 1-2$ y $Z \geq 2$ demuestran que, a lo sumo, hemos subvaluado la verdadera prevalencia de obesidad.

Por último, el tamaño de la muestra usado fue algo menor que el propuesto en el diseño; sin embargo, la diferencia fue pequeña y dependió también del tamaño del grupo de escolares con potenciales lazos de amistad.

\section{CONCLUSIÓN}

El estudio de las redes sociales de adolescentes permitió conocer las relaciones de afinidad entre aquellos jóvenes que presentan hábitos de consumo de tabaco o alcohol, o que padecen sobrepeso $\mathrm{u}$ obesidad. Tanto fumadores como bebedores parecen estar estrechamente enlazados en la red; mientras que aquellos con sobrepeso ocuparían una ubicación más marginal. La conformación de conglomerados favorece el contacto entre quienes tienen los mismos hábitos, lo cual refuerza la conducta, pero a la vez facilitaría la intervención sanitaria. El conocimiento de la conformación de las redes sociales y la intervención sobre solo algunos de sus actores podrían emplearse para propagar conductas saludables entre los adolescentes.

\section{BIBLIOGRAFÍA}

1. Van Jaarsveld CH, Fidler JA, Simon AE, Wardle J. Persisten impact of pubertal timing on trends in smoking, food choice, activity, and stress in adolescence. Psychosom Med 2007;69(8):798-806.

2. Melchior M, Chastang JM, Goldberg P, Fombonne E. High prevalence rate of tobacco, alcohol and drug use in adolescents and young adults in France: results from the GAZEL youth study. Addict Behav 2008;33:122-33.

3. Smalley SE, Wittler RR, Oliverson RH. Adolescent assessment of cardiovascular heart disease risk factor attitudes and habits. J Adolesc Health 2004;35:374-9.

4. Christakis NA, Fowler JH. The spread of obesity in a large social network over 32 years. N Engl J Med 2007;357:370-9.

5. Christakis NA, Fowler JH. The collective dynamics of smoking in a large social network. N Engl J Med 2008;358:2249-58.

6. Chen PH, White HR, Pandina RJ. Predictors of smoking cessation from adolescence into adulthood. Addict Behav 2001;26:517-29.

7. Powell LM, Tauras JA, Ross H. The importance of peer effects, cigarette prices and tobacco control policies for youth smoking behavior. J Health Econ 2005;24:950-68.

8. Girvan M, Newman MEJ. Community structure in social and biological networks. Proc Natl Acad Sci USA 2002; 99:8271-6.

9. Newman MEJ. The structure and function of complex networks. SIAM Review 2003;45:167-256. 
10. Bearman PS, Moody J, Stovel K. Chains of affection: the structure of adolescent romantic and sexual networks. $\mathrm{Am}$ J Sociol 2004;110:44-91.

11. Mulassi AH, Hadid C, Borracci RA, Labruna MC, et al. Hábitos de alimentación, actividad física, tabaquismo y consumo de alcohol en adolescentes escolarizados de la provincia y el conurbano bonaerense. Arch Argent Pediatr 2010;108(1):45-54.

12. Subcomisión de Epidemiología y Comité de Nutrición. Consenso sobre factores de riesgo de enfermedad cardiovascular en pediatría. Obesidad. Arch Argent Pediatr 2005;103(3):262-81.

13. Kamada T, Kawai S. An algorithm for drawing general undirected graphs. Information Processing Letters 1989;31:7-15.

14. Batagelj V, Mrvar A. Pajek - analysis and visualization of large networks. En: Junger M, Mutzel P, eds. Graph drawing software. Berlin: Springer; 2003. Págs. 77-103.

15. Kovalsky I, Bay L, Rausch Herscovici C, Berner E. Prevalencia de obesidad en una población de 10 a 19 años en la consulta pediátrica. Arch Argent Pediatr 2003;101(6):441-7.
16. Strauss RS, Pollack HA. Social marginalization of overweight children. Arch Pediatr Adolesc Med 2003;157:746-52.

17. Eisenberg D, Quinn BC. Estimating the effect of smoking cessation on weight gain: an instrumental variable approach. Health Serv Res 2006;41:2255-66.

18. McPherson M, Smith-Lovin L, Cook JM. Birds of a feather: homophily in social networks. Annu Rev Sociol 2001;27: 415-44.

19. Centola D, Eguiluz V, Macy M. Cascade dynamics of complex propagation. Physica A 2007;374:449-56.

20. Christakis NA. Social networks and collateral health effects. BMJ 2004;329:184-5.

21. Wing RR, Jeffery RW. Benefits of recruiting participants with friends and increasing social support for weight loss and maintenance. J Consult Clin Psychol 1999;97:132-8.

22. Pampel FC. Diffusion, cohort change, and social patterns of smoking. Soc Sci Res 2005;34:117-39.

23. Comité Nacional de Nutrición, Sociedad Argentina de Pediatría. Guías de práctica clínica para la prevención, el diagnóstico y el tratamiento de la obesidad. Arch Argent Pediatr 2011; 109(3):256-266. 
Apéndice

\section{DEFINICIONES DE LOS DISTINTOS PARÁMETROS PARA CONSTRUIR LAS REDES}

- Individuo o ego: es la persona cuya condición y comportamiento se quiere estudiar; en este caso, cada adolescente en su condición de fumador, bebedor u obeso.

- Amigo o alter: representa cada persona conectada al individuo en estudio, que podría influir sobre su conducta a través de la red social.

- Nodo: representa los objetos conectados o no a una red; en este caso cada nodo corresponde a un individuo o amigo.

- Lazo o conexión: es la forma gráfica de representar una relación entre dos nodos, en este caso, de amistad entre dos adolescentes.

- Grado de conexión: representa el número de conexiones de cada individuo en la red; en este caso, el número de amistades referidas por cada encuestado.

- Conglomerado (cluster): corresponde a un conjunto de nodos, cada uno conectado por lo menos a otro nodo dentro del mismo grupo.

- Homofilia (homophily): representa la tendencia de las personas a relacionarse con otros individuos con afinidades o atributos similares. Una forma de expresarla es a través de la probabilidad que tiene un individuo con cierto atributo de relacionarse con otras personas con los mismos atributos.

- Centralidad (centrality). Una vez construida la red, la centralidad mide cuan cerca están los individuos de su centro. Las personas con mayor número de conexiones ocupan un lugar más central en la red y viceversa. Por un lado, el grado de conexión de cada individuo es una buena medida de la centralidad; y por el otro, se puede calcular un coeficiente de centralidad (centrality-closeness) de cada individuo en la red, cuyos valores más altos indicarían un mayor grado de conectividad en la red y los más bajos un mayor aislamiento en ella.

- Componente gigante o conectado de la red (connected component). En las redes con un pequeño número de conexiones, los individuos suelen pertenecer a pequeñas islas de relación o amistad. A medida que la cantidad de conexiones aumenta, se genera un gran conjun- to de individuos conectados que se denomina componente gigante de la red. Cuanto mayor es el componente, mejor es la interconexión entre los adolescentes.

- Diámetro del componente gigante o conectado. La máxima separación entre dos adolescentes, también llamada diámetro del componente gigante, mide la distancia más grande que se debe recorrer para conectar dos individuos, en términos de cantidad de nodos interpuestos. Obviamente, este cálculo excluye a los individuos no conectados.

- Distancia o grados promedio de separación. Para un individuo dado, sus amistades tendrán un grado de separación igual a 1, mientras que las amistades de sus amistades tendrán un grado de separación igual a 2 con el individuo original, y así sucesivamente. A partir del cálculo de la distancia mínima entre todos los pares de adolescentes de la red, se obtiene la distancia promedio de separación para el conjunto de los individuos conectados.

- Clustering: La mayoría de los individuos en una red social se asocian en conglomerados (clusters) de comunidades con afinidades similares; en el caso de los adolescentes, podrían existir conglomerados de amistades separadas por sexo o hábitos de consumo. Para probar la formación de conglomerados se recurre al cálculo del coeficiente $C$ de aglomeración, de acuerdo a la siguiente expresión:

$C=3 \times$ número de triángulos en la red/ número de tripletes de vértices conectados

Donde "triángulo" corresponde a un trío de amigos todos conectados entre sí, y un "triplete conectado" es un único individuo conectado a otros dos. Así, el coeficiente $C$ vale 1 cuando existe una red completamente conectada (todos los individuos se conectan con todos); mientras que si las conexiones son al azar, o sea, si no conforman un conglomerado específico, el coeficiente $C$ se acercará al valor cero $(1 / \mathrm{N}$, siendo $\mathrm{N}$ el número de nodos de la red), como en el caso de una red generada al azar. 


\section{Anexo electrónico. Estructura de la encuesta}

\section{REDES EN ADOLESCENTES ESCOLARIZADOS}

\section{DATOS GENERALES}

$\mathrm{N}^{\circ}$ asignado al encuestado:.

Fecha:.../.../...

Colegio:.

\section{DATOS PERSONALES}

Apellido:

Edad:.... años...

Domicilio particular:

Calle:

Año que cursa:.........

Peso:...... kg. Altura:..... cm.

TA:...........mmHg.

\section{MEJORES VÍNCULOS CON COMPAÑEROS}

En el siguiente cuadro escribí los Nombres y Apellidos de los alumnos que concurran a tu colegio con quienes tengas mejores relaciones de amistad o compañerismo. Citarlos en orden de importancia.

No escribir en la columna de $\mathrm{N}^{\circ}$ adjudicado; el encuestador lo realizará.

Nombre y Apellido del compañero con mejores vínculos

Teléfono:

Horas de escolaridad:....h.

Perímetro de cintura:......cm.
$\mathrm{N}^{\circ}$ adjudicado

(Permite incluir hasta 10 amistades)

\section{ALIMENTOS}

¿Consumís golosinas en el colegio?: Sí $\square$ No $\square$

¿Consumís sándwiches o hamburguesas o panchos en el colegio?: Sí $\square$ No $\square$

¿Lo hacés con frecuencia en compañía de alguna de las personas que citaste como mejor amigo o compañero? Sí $\square$ No $\square$ ¿Te cuidás para mantener o bajar de peso? Sí $\square$ No $\square$

\section{TABAQUISMO}

¿Fumaste alguna vez en tu vida?:

¿Fumaste en los últimos seis meses?:

Sí $\square$ No $\square$

Sí $\square$ No $\square$

$\begin{array}{ll}\text { ¿Fumás diariamente?: } & \text { Sí } \square \text { No } \square \\ \text { Si fumás, ¿lo hacés para bajar de peso?: } & \text { Sí } \square \text { No } \square\end{array}$

\section{ACTIVIDAD FÍSICA}

¿Realizás deportes? (ya sea para competir o no): $\quad$ Sí $\square$ No $\square$

¿Qué deporte realizás con mayor frecuencia?:

Fútbol $\square \quad$ Tenis $\square \quad$ Ciclismo $\square \quad$ Hockey $\square \quad$ Otros $\square$

¿Cuántas veces por semana?: 1 o 2 veces $\square \quad 3$ o más $\square$

\section{TIEMPO LIBRE}

¿En qué ocupás generalmente el tiempo libre?:

Mirando TV

Con videojuegos

Leyendo

Practicando juegos o deporte

\section{BEBIDAS ALCOHÓLICAS}

¿Consumís bebidas que contengan alcohol?: Sí $\square$ No $\square$

¿Cuántas veces al año consumís bebidas con alcohol (cerveza, vino, champaña, whisky, fernet, tequila, vodka, etc.)?:
1 vez $\square$
2 veces
3 veces o más $\square$

¿Dónde consumís alcohol con más frecuencia?:

En el boliche $\square \quad$ Bar $\square \quad$ Casa/s de amigo/s $\square \quad$ En tu casa $\square$ 